\title{
ASRSM: A Sequential Experimental Design for Response Surface Optimization
}

\author{
Adel Alaeddini, ${ }^{a * \dagger}$ Kai Yang ${ }^{b}$ and Alper Murat ${ }^{b}$
}

\begin{abstract}
Most preset response surface methodology (RSM) designs offer ease of implementation and good performance over a wide range of process and design optimization applications. These designs often lack the ability to adapt the design on the basis of the characteristics of application and experimental space so as to reduce the number of experiments necessary. Hence, they are not cost-effective for applications where the cost of experimentation is high or when the experimentation resources are limited. In this paper, we present an adaptive sequential response surface methodology (ASRSM) for industrial experiments with high experimentation cost, limited experimental resources, and high design optimization performance requirement. The proposed approach is a sequential adaptive experimentation approach that combines concepts from nonlinear optimization, design of experiments, and response surface optimization. The ASRSM uses the information gained from the previous experiments to design the subsequent experiment by simultaneously reducing the region of interest and identifying factor combinations for new experiments. Its major advantage is the experimentation efficiency such that for a given response target, it identifies the input factor combination (or containing region) in less number of experiments than the classical single-shot RSM designs. Through extensive simulated experiments and real-world case studies, we show that the proposed ASRSM method outperforms the popular central composite design method and compares favorably with optimal designs. Copyright $\odot 2012$ John Wiley \& Sons, Ltd.
\end{abstract}

Keywords: adaptive sequential experiment; design of experiments; central composite design (CCD); optimal designs; response surface optimization

\section{Introduction}

$\mathrm{M}$ ost process and design optimization approaches such as the response surface methodology (RSM) require a complete experimental design to be determined prior to the experimentation process. ${ }^{1}$ These preset designs offer ease of implementation and good performance over a wide range of applications. However, they lack the ability to adapt the design on the basis of the characteristics of application and experimental space so as to reduce the number of experiments necessary. This, in particular, constitutes a major disadvantage in many industrial applications where the cost of experimentation is high or when the experimentation resources are limited. These industrial experiments share the following two main characteristics: (i) prior to the experiment, the behavior of the experimental design space is not well known, and (ii) the cost of each experimental trial is prohibitively high, and the experimental budget is limited. An example for such industrial experiments is the combustion test for aircraft engine or turbines where prototypes are very expensive and the behavior of different designs are highly unpredictable (Figure 1). The computational experimentation approach commonly resort as a cost-effective alternative to physical testing of complex engineering systems. However, these computational experiments may take 5 to $20 \mathrm{~h}$ per simulation run. ${ }^{2} \mathrm{Gu}^{3}$ reported that one crash simulation on a full passenger car takes 36-160 $\mathrm{h}$ at Ford Motor Company.

The focus of this paper is on the industrial experiments with high experimentation cost, limited experimental resources, and high design optimization performance requirement. In designing industrial experiments, the traditional RSM methodologies (central composite design (CCD), Box-Behnken optimal designs, etc.) are often preferred for various advantages, for example, rotatability and variance of error estimation. However, these methods rely on 'one-shot' designs and thus fall short in providing efficient experimental designs for highly engineered complex systems. This has been pointed out by George E. P. Box, 'There should be more studies of statistics from the dynamic point of view' in Box ${ }^{4}$ and 'I think we have spent too much time on one-shot statistical procedures designed to test rather than to learn' in response to Myers. ${ }^{5}$ Further, these methods fit a regression model of the system responses to accurately predict the response curve over the entire domain of feasibility. However, the prediction in the neighborhood of the optimum is often more important than the prediction in the domain of feasibility.

${ }^{a}$ University of Michigan, Industrial and Operations Engineering, Ann Arbor, MI, USA

${ }^{b}$ Wayne State University, Industrial and Systems Engineering, Detroit, MI, USA

*Correspondence to: Adel Alaeddini, University of Michigan, Industrial and Operations Engineering, Ann Arbor, MI, USA.

${ }^{\dagger}$ E-mail: alaedini@umich.edu 


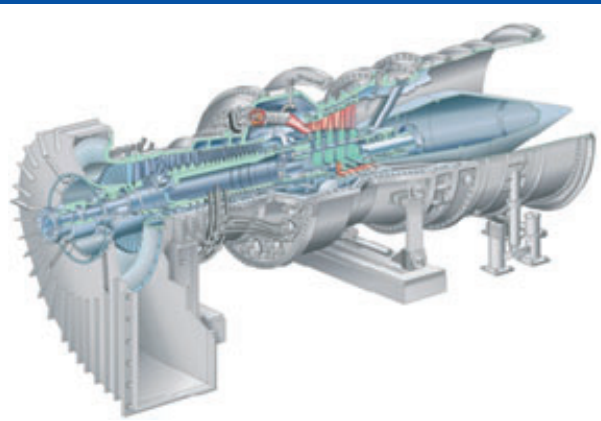

(a) Turbine

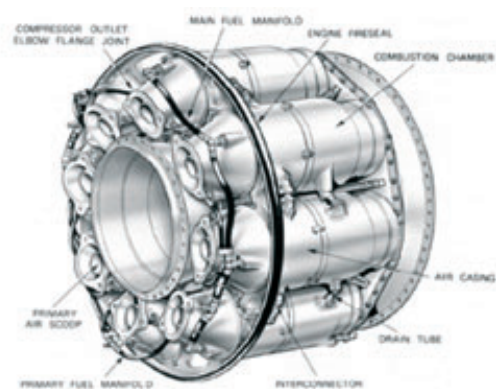

(b) Combustion Chamber

Figure 1. Turbine and aircraft engine

In this study, we propose an adaptive sequential experimentation approach on the basis of an adaptive sequential response surface methodology (ARSRM) for optimizing design and processes. We consider the experimentation as a successive series of small data collection efforts. At each step, we learn from the previous results, refine our understanding, and develop a new model for the next experiment to reduce uninformative experiments and improve the quality of results. The idea of adaptive experimental design is not new. Beginning with the sequential RSM experimentation with multiple blocks in Box and Wilson, ${ }^{6}$ there have been many ideas such as the one-factor-at-a-time (OFAT), ${ }^{7,8}$, adaptive OFAT, ${ }^{9}$, adaptive RSM, ${ }^{10,11}$ successive RSM, ${ }^{12}$ evolutionary operation, ${ }^{13}$ steepest ascent based methods, ${ }^{6}$ and sequential and adaptive approximation methods from the engineering design discipline.

The ARSRM approach presented in this paper is a local optimization approach for physical experiments where the region of interest of two primary input factors is already determined. The shifting the region of interest close to the optimum is beyond the scope of this paper and is assumed to be performed a priori using an efficient method such as the steepest descent. Furthermore, in most practical applications, the current settings of the factor values are usually determined and known to produce a stable response and a satisfactory yield. However, because of extraneous changes over time, the current conditions may become less robust and suboptimal. Hence, there might be easy gains in yield by moving in the surrounding region of the design space. In addition to knowing the two primary input factors and their advantageous ranges, we also assume that a second-order model is most reasonable to characterize the underlying relationship between the response and the input factors. We do not make any assumptions regarding the noise in response. Hence, our goal is to precisely estimate the relationship between important factors and response and identify the most likely location for the process/product to be optimized in the detailed RSM experimentation stage.

The most salient aspect of the proposed ARSRM approach is its experimentation efficiency. Specifically, the proposed approach identifies, for a given response target, the input factor combination (or containing region) in less number of experiments than the classical single-shot RSM designs. This sequential adaptive approach uses the information gained from the previous experiments to design the subsequent experiment by simultaneously reducing the region of interest and identifying factor combinations for new experiments. This reduction is achieved through rank ordering of the responses of preceding experiments. Throughout the process, we consider a fixed design (i.e., full factorial and center point) that allows inheriting some of the experiments from the previous runs. As a result, this method efficiently increases the accuracy and precision of the estimated optimal point by reducing the region of interest.

This paper differs from earlier approaches on adaptive and sequential RSM in three different ways. First, the reduction of the region of interest is optimal if the relationship between the response and input factors is quadratic and the response is deterministic. Specifically, the optimal factor combination is always contained in the reduced region. Second, the ARSRM method requires fewer experiments in each run as a result of inheriting previous experiments and fixed design structure. Lastly, the proposed ARSRM method identifies the reduced region of interest with a ranking-based method rather than the response levels obtained from each experiment. This is indeed similar to using not the value of a parameter but its rank in robust statistics. ${ }^{14}$

In what follows, we briefly review the literature on advancements in RSM with special emphasis on the adaptive experimentation methodologies in Section 2. Section 3 presents the proposed ASRSM methodology in detail. In Section 4, we present the results of applying the ASRSM method to the real-world experiments. Section 5 presents the performance results of the ASRSM method compared with the classical CCD RSM method and three optimal designs. Finally, Section 5 discusses the results and presents future research directions.

\section{Response surface methodology literature}

This section presents the relevant literature for the proposed adaptive experimental design methodology. We first review the classical response surface methodologies and then the more advanced methods including optimal design. Finally, we briefly describe other adaptive design methodologies such as steepest ascent, simplex-based methods, evolution operation methods, adaptive OFAT methods, adaptive RSM, and successive RSM. 
The RSM has been used as one of the most effective tools for process and product development since its introduction by Box and Wilson. ${ }^{6}$ RSM consists of statistical and numerical/mathematical optimization techniques for examining the relationship between one or more response variables and a set of quantitative experimental variables or factors. Because the literature on RSM is vast, we herein refer the reader to a number of good review studies such as Box, ${ }_{1}^{4}$ Myers, ${ }^{5}$ and Myers et al. ${ }^{15,16}$ CCD and Box-Behnken design are the most popular classes of designs used for fitting second-order model. ${ }^{17}$ Generally, the CCD with $k$ factors consists of a $2^{k}$ factorial runs augmented with $2^{k}$ axial or star runs and at least one center run. There are usually two parameters in CCD that must be specified: the distance $\alpha$ of the axial runs from the design center and the number of center points. Three to five runs are recommended in the literature. ${ }^{18}$ The number of runs in CCD increases exponentially with the number of design variables and hence becomes inefficient for high dimensional design problems.

Optimal design methodologies select designs that are 'best' with respect to some criterion. This selection process includes specifying the model, determining the region of interest, selecting the number of runs to make, specifying the optimality criterion, and choosing the design points from a set of candidate points spaced over the feasible design region. Kiefer ${ }^{19,20}$ and Kiefer and Wolfowitz ${ }^{21}$ greatly contributed to the development of the idea of optimal designs. D-optimal design is the most widely used criterion in optimal designs. A design is said to be D-optimal if $\left|\left(X^{\prime} X\right)^{-1}\right|$ is minimized, where $X$ is the design matrix with the number of rows and the number of columns equal to the number of experiments in the design and the number of coefficients in the response model, respectively. Note that this is equivalent to minimizing the volume of the joint confidence region of the vector of regression coefficients. There are also other types of optimal designs such as the V-optimal design that deals with only the variance of the regression coefficients and the V-optimal design that minimizes the average prediction variance over the set of $m$ points of interest. Pukelsheim ${ }^{22}$ provides an excellent source on the optimal design of experiments.

The idea of sequential and adaptive experimental design is not new. Box and Wilson ${ }^{6}$ suggest a two-stage sequential CCD where the first stage is a two-level factorial or fractional factorial design and the axial points constitute the second stage. The method of steepest ascent ${ }^{6}$ is another adaptive sequential experimentation approach in which the experimental points move sequentially along the gradient direction. Evolutionary operation, another adaptive experimental approach, iteratively builds a response surface around the optimum from the previous iteration by drifting factorial experiments with center points. ${ }^{13,23}$ These approaches are primarily used for shifting the region of interest close to the optimum and replicate the same experimental design iteratively in different regions of the factor space.

OFAT can be considered as the earliest adaptive sequential experimentation approach proposed. ${ }^{7}$ OFAT changes one variable at a time while keeping others constant at fixed values to find the best response. Once a factor is changed, its value is fixed in the remainder of the process. This process is repeated until all the variables are tried. We refer the reader to Box et al., ${ }^{24}$ Montgomery, ${ }^{18}$ and Czitrom $^{25}$ for more detailed discussion on OFAT. Frey et al. ${ }^{9}$ introduced adaptive one-factor-at-a-time (AOFAT) experimentation method. They compared the AOFAT technique with orthogonal arrays through computer simulations and concluded that AOFAT technique tends to achieve greater gains than those of orthogonal arrays when experimental error is small or the interactions among control factors are large. Frey and Jugulum ${ }^{26}$ investigated the mechanisms by which AOFAT technique led to an improvement. Frey and Wang ${ }^{27}$ presented the models of AOFAT and factor effects and illustrated with theorems that AOFAT method exploits main effects if interactions are small and exploits two-factor interactions when two-factor interactions are large.

Wang et al. ${ }^{10}$ developed an adaptive RSM methodology, called adaptive response surface method (ARSM). ARSM is a sequential experimentation method, where, at each iteration, it discards portions of the design space that correspond to the response values worse than a given threshold value. Such elimination reduces the design space gradually to the neighborhood of the global design optimum. ARSM performs a CCD experiment at each iteration, and thus, the number of required design experiments increases exponentially with the number of design variables. Further, ARSM does not inherit any of the previous runs and requires a completely new set of CCD points. Wang ${ }^{11}$ proposed a modified ARSM where the CCD is substituted with Latin hypercube design. Stander ${ }^{12}$ proposed the successive RSM method that uses a region of interest, a subspace of the design space, to determine an approximate optimum. A range is chosen for each variable to determine its initial size. Then, a new region of interest is centrally built on each successive optimum. The improvement in response is attained by moving the center of the region of interest as well as reducing its size through panning and zooming operations, respectively. At each subregion, a D-optimal experimental design is used to best utilize the number of available runs together with over-sampling to maximize the predictive capability.

\section{Proposed methodology}

The proposed ASRSM methodology is developed on the basis of nonlinear and numerical optimization, design of experiments, and response surface optimization. In this section, we discuss the detailed elements of each run of the ASRSM. We first describe the terminology and state the assumptions of ASRSM in Section 3.1. Next we provide an overview of the methodology in Section 3.2 and then describe the two core strategies embedded in ASRSM: (i) parametric approach in Section 3.3 and (ii) non-parametric approach in Section 3.4. In Section 3.5, we describe how these two strategies are integrated within ASRSM.

\subsection{Terminology and assumptions}

The definitions and terminology used in the proposed ASRSM methodology is as follows. Some of the notations are illustrated in Figure 2 for two-dimensional factor space with five experiments in each run: 


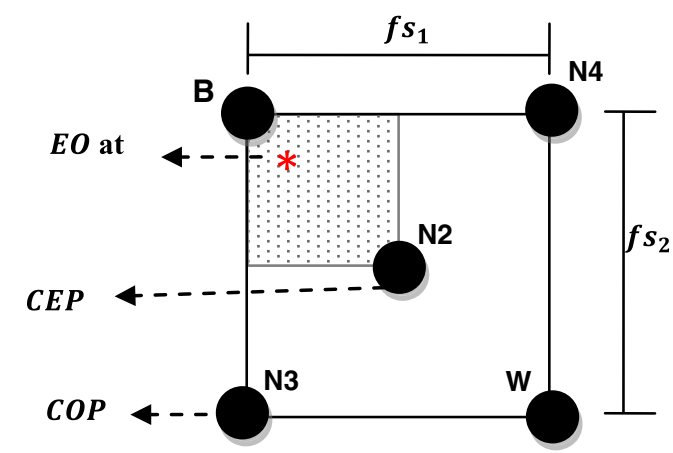

Legend

$F S$ of $1^{\text {st }}$ run

$O R$ of $1^{\text {st }}$ run

Figure 2. An illustration of terminology on a two-dimensional factor space with $e=5$

$F S_{r} \quad$ Factor space at run $r$ and expressed as Cartesian product of factor ranges in run $r$

$\mathrm{fs}_{i} \quad$ Initial range of factor $i$

COP A corner point experiment run at the intersection of extrema of factor ranges

CEP Center point experiment run at the center of gravity of the factor space

$r \quad$ Index of runs, for example, $r=1,2, \ldots, R$, where $R$ is the total number of runs

$e \quad$ Index of experiments in a given run, for example, $e=1,2, \ldots, E$, where $E$ is the total number of experiments

$B \quad$ The experiment with the best response level in a given run

$N_{k} \quad$ The experiment with the $k$ th best response level in a given run $(2 \leq k \leq e-1)$

$W \quad$ The experiment with the worst response level in a given run

$O R \quad$ Optimal region in run $r$ containing the estimated optimal experiment, $O R \quad F S_{r}$

$O$ Optimal experiment, for example, best experiment in the initial factor space

$E O_{r} \quad$ Estimated optimal experiment in run $r$, for example, best incumbent estimation of the optimal experiment

$B C E \quad$ Best at center classification of the $O R_{r}$ where the location of $B$ is at CEP

$B C O$ Best at corner classification of the $O R_{r}$ where the location of $B$ is at the corner of factor space

As in most RSM approaches, the proposed ASRSM methodology relies on a number of simplifying assumptions. The extensions due to the relaxation of these assumptions are beyond the scope of this paper, and some of these extensions are discussed in the conclusion. For the proposed methodology, we consider the following assumptions:

1. There are two significant and controllable factors.

2. The underlying relation between a single response and two factors can be represented by a quadratic model. RSM models are usually employed in a sufficiently small region around the optimal region. As a result, it is quite common in RSM applications to assume that the underlying model can be approximated via a quadratic function. Such assumption also holds for this study. The response is convex in the region of interest. We assume that the region of interest is shifted close to the optimum a priori using an efficient method (e.g., steepest descent). Because for most of the nonlinear minimization problems the underlying model is locally convex around the optimal solution, we assume that the response is convex in the region of interest. Our empirical test results show that the proposed approach is robust with respect to this assumption such that the proposed method is effective in cases where the underlying model is non-convex.

3. The factor space in the region of interest is feasible.

\subsection{Algorithm and initial run design}

Figure 3 illustrates the structure of the proposed ASRSM methodology. The procedure is initialized with a region of interest, for example, a feasible factor space that is guaranteed to contain the $O$. The goal is to reach to the vicinity of $O$ with a finite set of runs $(R)$. Each run $r$ is set up on a given factor space $\left(F S_{r}\right)$ with a specific experimental design $(D)$, for example, a modified version of the factorial design augmented with a center point. The experiments in each run $r$ are taken one at a time, and the $F S_{r}$ is not finalized until all experiments are taken. Once an experiment $e$ is taken, the $E O_{r}$ is obtained from the parametric model fitting using all the experiments in all runs. For all but last experiment in run $r$ (i.e., $e \neq E$ ), the $E O_{r}$ is tested for belonging to $F S_{r}$. Accordingly, $F S_{r}$ is updated (e.g., expanded) if $E O_{r}$ is outside $F S_{r}$. For the last experiment (i.e., $e=E$ ), the approach follows two concurrent strategies, for example, non-parametric ranking strategy and parametric model fitting strategy. According to the ranking of experiments and $E O_{r}$ from the quadratic model fitting, a reduced factor space containing the $E O_{r}$ (i.e., $O R_{r}$ ) is determined for the next run. This procedure continues until the convergence criteria based on estimated optimal experiment or adjusted coefficient of determination of the fitted model is attained. In particular, the convergence criteria are met if the adjusted coefficient of determination of the fitted quadratic model ( $\left.R_{\text {adj }}^{2}\right)$ exceeds a preset threshold value $\left(\theta R_{\text {adj }}^{2}\right)$, or the rate of change in the coefficient of determination $\left(\mid \Delta R_{\text {adj }}^{2}\right)$ or in the estimated optimal experiment $(\triangle(E O))$ because the previous run is below preset threshold values $\delta R_{\text {adj }}^{2}$ and $\delta_{\mathrm{EO}}$, respectively. The motivation for the dual 


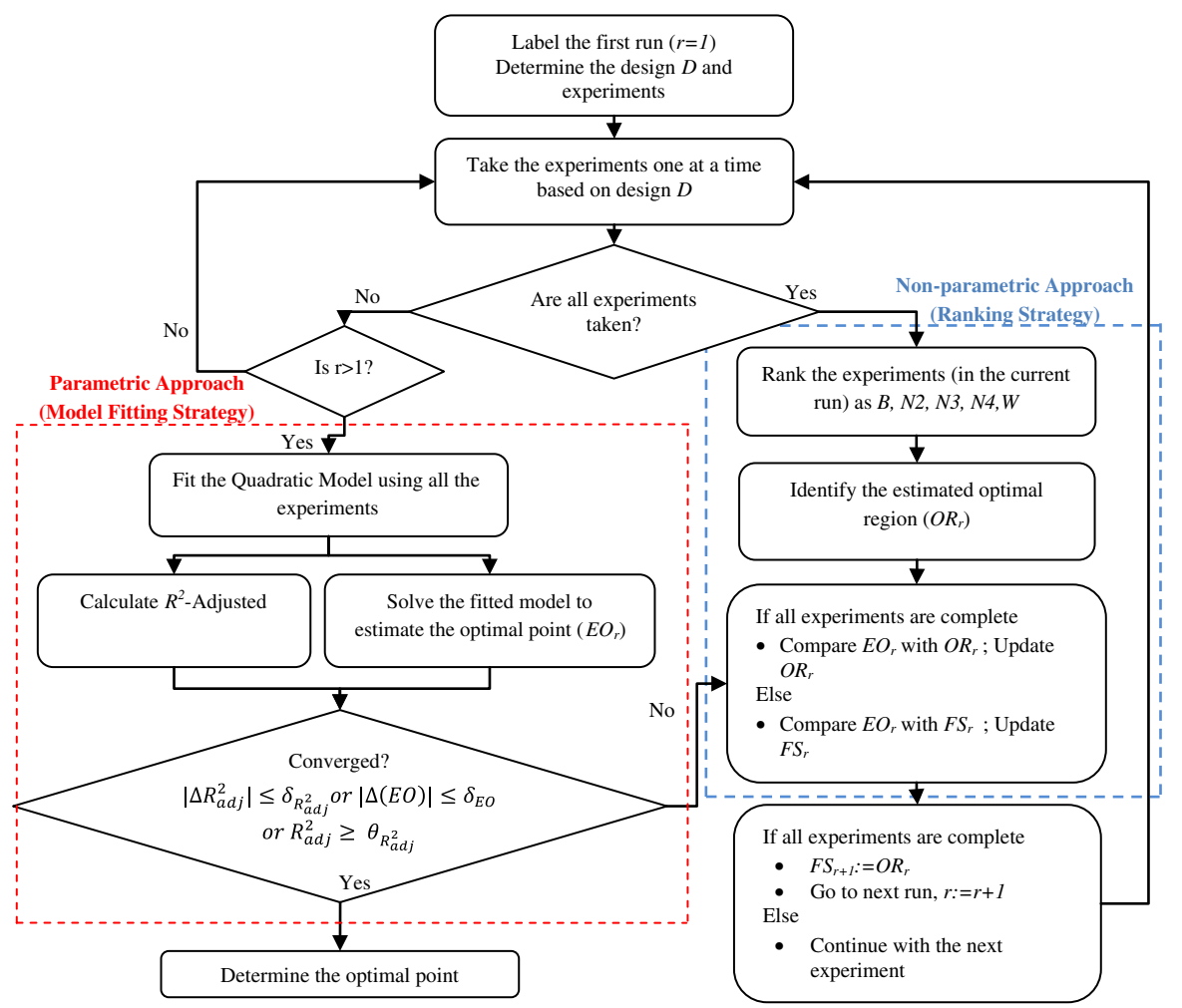

Figure 3. Scheme of the proposed adaptive sequential response surface methodology

strategy (e.g., parametric versus non-parametric) is that while the information from the ranking strategy is accurate but not precise, the information from the model fitting is precise but not accurate.

The factor space of each run $\left(F S_{r}\right)$ can be expressed as a mapping $\left(\varphi_{r}\right)$ of the factor space of the preceding run $\left(F S_{r-1}\right)$. In most general form, the proposed methodology generates a series of factor spaces that are nested, for example, $F S_{r}=\varphi_{r}\left(\varphi_{r-1}\left(\cdots \varphi_{0}\left(F S_{1}\right)\right)\right)$. The output of this mapping $\varphi_{r}$ depends on the current factor space, the experimentation design $(D)$, the outcome of ranking of experiments as well as the result of parametric strategy described in the next subsection. The latter two, the ranking and the parametric strategies, are described in Sections 3.3 and 3.4, respectively. Before discussing the $D$ used in each run and the initial factor space, we briefly present the algorithm using the illustration in Figure 4 for a special case. The proposed approach is initialized with $F S_{1}$, and the indicated five experiments $(B, N 2, N 3, N 4, W)$ are taken from the corresponding design $D$. Once the responses are ranked, the non-parametric ranking strategy identifies the $O R_{1}$. Next, the parametric model fitting approach determines the $E O_{1}$ using the first five experiments. Lastly, the $E O_{1}$ and $E O_{1}$ are compared to determine the new factor space $\left(F S_{2}\right)$. Note that the design in $r=2$ inherits two experiments from the first run, namely $B$ and $N 2$.

The proposed ASRSM method uses the same $D$ in each run that is the factorial design augmented with a center point. Hence, we maintain the same experimental design $D$ and consider a constant number of experiments (e.g., $E=5$ ) throughout the process. In practice, none of the existing methods for setting the initial point in sequential optimization procedures is superior to the corner

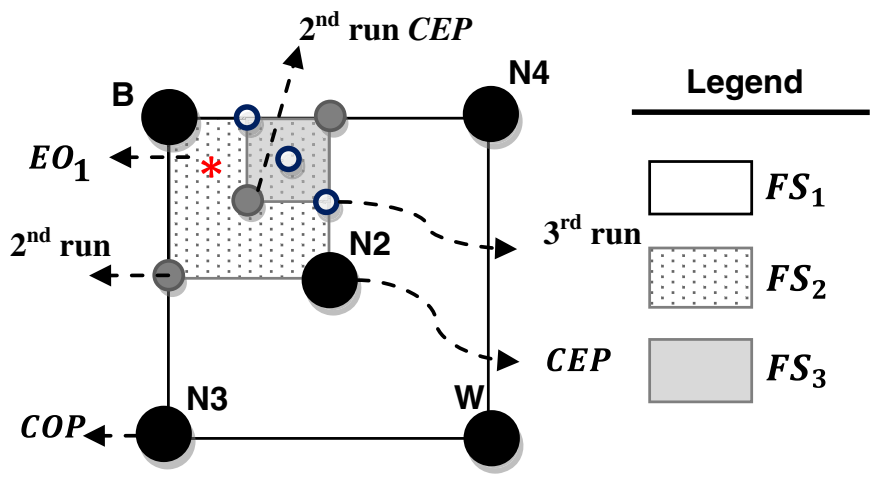

Figure 4. Illustration of the factor space reduction across runs $r=1,2$, and 3 
initial point as in the factorial design. ${ }^{31}$ Furthermore, the experiments conducted on corner points benefit from fractional factorial design, especially when the design is orthogonal. In particular, the designs maximize the amount of information gained from each experiment. On the other hand, central points are essential for modeling the curvature of the underlying function. ${ }^{18}$ Lastly, the five experiments in $r=1$ are not sufficient to estimate the full quadratic response model. Hence, we estimate the $E O_{1}$ by fitting a quadratic response model without the constant term.

The design of the initial factor space in the proposed approach is adapted such that a rational comparison with traditional RSM methods (e.g., CCD) is possible. In the traditional CCD approach, the corner points are taken at \pm 1 unit distance from the center point $(0,0)$. In comparison, the proposed methodology starts with a broader initial region around the center point, for example, at $\pm \sqrt{2}$ unit distance from the center. Figure 5(a) illustrates the initial factor space of the traditional CCD and the proposed method with light and dark experiment points, respectively. Although beginning with a larger space is initially disadvantageous, experimental results demonstrate that the reduction in the factor space with the same number of experiments far exceeds initial difference. An additional benefit is that this modification may decrease the effect of random error on the initial results. Let us consider the diagonal cross section of these two designs as illustrated in Figure $5(\mathrm{~b})$ and assume that the noise is identically distributed on this cross section. Then, it can be shown that the impact of the noise on the prediction of the optimal experiment point is less with the proposed methodology's factor space.

\subsection{Non-parametric approach: ranking strategy}

At each run $r$ of the proposed ASRSM approach, we first rank the five experiments (e.g., four factorial and one center) as $B, N 1, N 3$ and $W$ according to their response levels. On the basis of the ranking, we identify the implied optimal region that contains the $E O_{r}$. This region is a polygon contained in $F S_{r}$ and can be convex or non-convex in the space of factors. We then identify a rectangle that contains the implied optimal region and denote it as the optimal region $\left(O R_{r}\right)$, which determines the factor space of the next run.

This process of encapsulating the implied optimal region with a rectangle is a form of relaxation and is not efficient in terms of factor space reduction. However, there are valid reasons that motivate this relaxation. The foremost reason is the reduced need for new experiments because of the inheritance of experiments from the previous run. Secondly, the rectangular FS preserves the orthogonality of factorial experimental design. Further, this rectangular form facilitates the recursive characterization of the same rectangular structure throughout the process. In addition, we can use the same experimental design structure, for example, full factorial with a center point. Specifically, with rectangular envelope, the mapping across runs will be identical, for example, $\varphi(\cdot) \equiv \varphi_{r}(\cdot)$ for $\forall r$. This is because we maintain the same experiment design structure, and there is a finite number of optimal regions as a result of ranking outcomes. Lastly, the relaxation reduces the risk of selecting an optimal region that excludes the optimal experiment.

An alternative to the rectangular envelope is the convex hull of implied optimal region. Because of its convexity, it also allows for easier tessellation of the FS. While the convex hull reduces the optimal region more than the rectangular envelope, it does not reduce the number of new experiments as much. Furthermore, the experimental design used in each run will be different because the convex hulls of the implied optimal regions will vary in shape. Clearly the choice of the right form is a trade-off between the rate of contraction of the optimal region and the total number of experiments conducted. To better illustrate this trade-off, let us consider the implied optimal region in Figure 6(a). The convex hull of this implied optimal region is identified in Figure $6(\mathrm{~b})$ with six vertices (corner points). In contrast, we adopted the rectangular envelope that is illustrated in Figure 6(c). Comparison between Figure 6(b) and 6 (c) reveals that while convex hull based $O R$ leads to the greatest factor space reduction, it also leads to an increased number of new experiments (seven versus three new experiments) and cannot inherit experiments from previous runs. Note that it is not practical to change the design and choose only three new experiments (e.g., two vertices and one at the center of gravity) for the

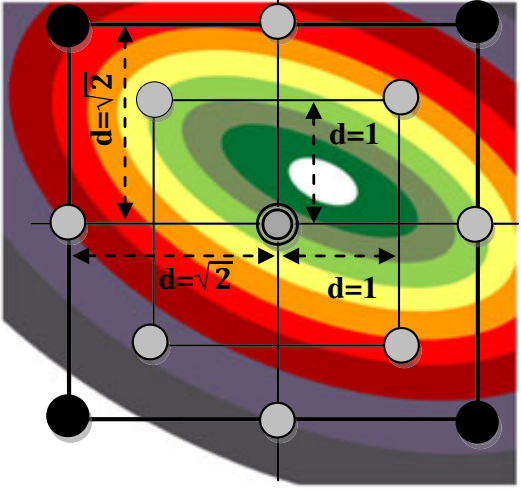

(a)

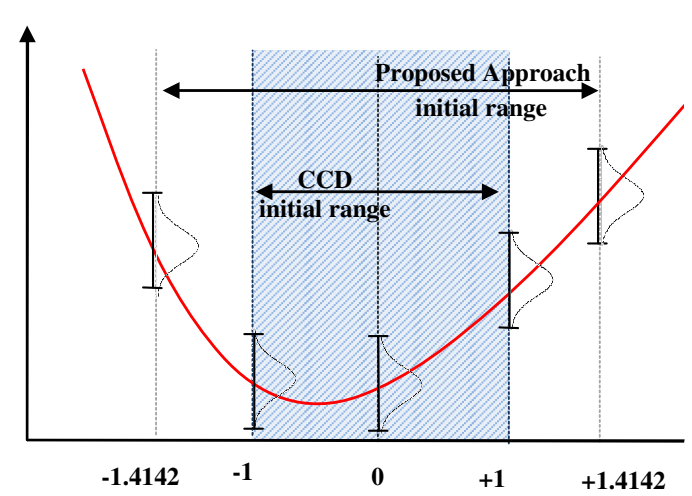

(b)

Figure 5. (a) Initial factor space and design structure and (b) diagonal cross section of the traditional central composite design and proposed adaptive sequential response surface methodology approach 


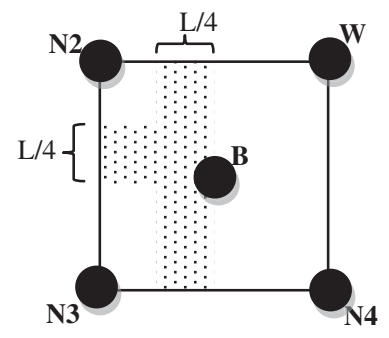

(a)

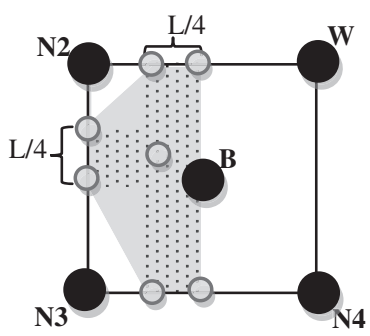

(b)

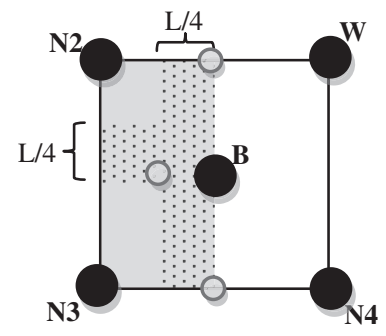

(c)

Figure 6. (a) Implied optimal region, (b) convex hull envelope of the implied optimal region, and (c) rectangular envelope of the implied optimal region

convex hull in Figure $6(\mathrm{~b})$. This is because we assume that the $O$ is contained in the current factor space, and, by choosing fewer number of vertices, we would then be implicitly reducing the implied optimal region.

In what follows, we present the optimal region alternatives on basis of the ranking of the experiments and the location of $B$ in the current FS.

3.3.1. Best at center $(B C E)$ optimal regions. An important information obtained from the ranking of experiments is the location of $B$. When $B$ is located at the center, the current $F S$ is then classified as having a BCE optimal region. Depending on the location of N2, N2, $N 4$, and $W$, there are three possible ORs as illustrated in Figure 7. We first determine the implied optimal region, illustrated as dotted regions in Figure 7. Next, we characterize the $O R$ as the rectangle that contains this implied optimal region.

The implied optimal regions are guaranteed to contain the optimal experiment in the absence of random noise. The mathematical proofs of the optimality of these implied optimal regions is involved and thus excluded. Instead, we provide a general proof sketch of the rectangular optimal regions and illustrate it for the $O R$ in Figure 7(a). The proofs are accomplished through the following steps: (i) Divide the non-optimal region into smaller rectangular subregions using factor centerlines; (ii) assume that the optimal point falls in one of these subregions; (iii) relocate the origin to that region and formulate the responses at $B, N_{1}, \ldots$, and $W$ on the basis of their displacement from the new origin; (iv) show that at least one pairwise comparison of the responses violates the initial ranking; and (v) replicate steps (ii)-(v) until all subregions are evaluated. The proof of $O R$ in Figure $7(a)$ is as follows.

\section{Proposition}

For a $B C E$ optimal region with ranking in Figure $7(\mathrm{a})$, the optimal experiment is located in the optimal region characterized as the quadrant with corners at $B$ and $N 2$ when there is no random noise.

$$
\begin{aligned}
\mathrm{d} z_{N 2 N 4}= & z_{N 2}-z_{N 4} \\
= & A\left[\left(\mathrm{~d} x_{2}\right)^{2}-\left(\mathrm{d} x_{4}\right)^{2}\right]+B\left[\left(\mathrm{~d} y_{2}\right)^{2}-\left(\mathrm{d} y_{4}\right)^{2}\right]+C\left[\left(\mathrm{~d} x_{2}\right)\left(\mathrm{d} y_{2}\right)-\left(\mathrm{d} x_{4}\right)\left(\mathrm{d} y_{4}\right)\right] \\
\mathrm{d} z_{N 3 N 4}= & z_{N 3}-z_{N 4}=A\left[\left(\mathrm{~d} x_{3}\right)^{2}-\left(\mathrm{d} x_{4}\right)^{2}\right]+B\left[\left(\mathrm{~d} y_{3}\right)^{2}-\left(\mathrm{d} y_{4}\right)^{2}\right] \\
& +C\left[\left(\mathrm{~d} x_{3}\right)\left(\mathrm{d} y_{3}\right)-\left(\mathrm{d} x_{4}\right)\left(\mathrm{d} y_{4}\right)\right] .
\end{aligned}
$$

Because the response is convex (e.g., $A, B>0$ ), we consider three response scenarios: $C=0, C<0$, and $C>0$. Note that when $O$ is in II, we have $\left|\mathrm{d} x_{2}\right|>\left|\mathrm{d} x_{4}\right|, \mathrm{d} x_{2} \leq 0$, and $\mathrm{d} y_{2}=\mathrm{d} y_{4}$, making the second term $\mathrm{d} z_{N 2 N 4}$ zero. For $C=0$, we have the first term in $\mathrm{d} z_{N 2 N 4}$ positive; thus, $\mathrm{d} z_{N 2 N 4}>0$, which is a contradiction to the ranking $z_{N 2}<z_{N 4}$. For $C<0$, the third term in $\mathrm{d} z_{N 2 N 4}$ is positive because $\mathrm{d} x_{2} \leq 0$, thus making $\mathrm{d} z_{N 2 N 4}>0$, which is also a contradiction. Lastly, for $C>0$, the first and second terms in $\mathrm{d} z_{N 3 N 4}$ are positive because $\left|\mathrm{d} x_{3}\right|>\left|\mathrm{d} x_{4}\right|$ and $\left|d y_{3}\right|>\left|d y_{4}\right|$. The last term in $d z_{N 3 N 4}$ is also positive because $d x_{3} d y_{3}>0$ and $\left|d x_{3} d y_{3}\right|>\left|d x_{4} d y_{4}\right|$. Thus, d $z_{N 3 N 4}>0$, which is a

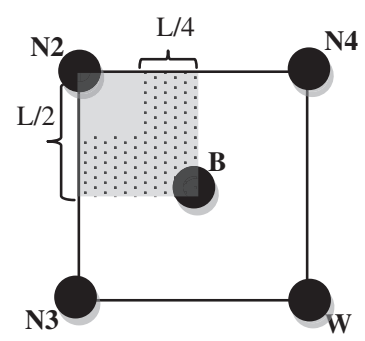

(a) Central Design with N2 cornered with N3 and N4

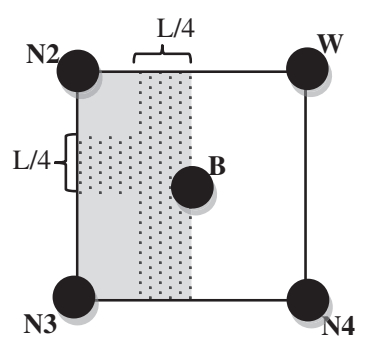

(b) Central Design with N2 cornered with $\mathrm{N} 3$ and $\mathrm{W}$

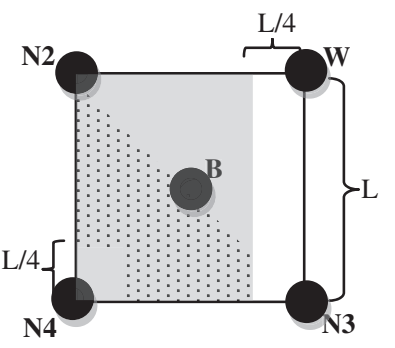

(c) Central Design with N2 cornered with $\mathrm{N} 4$ and W

Figure 7. $B C E$ ORs (dotted region, implied $O R$; shaded region, $O R$ ) 
contradiction to the ranking $z_{N 3}<z_{N 4}$. For the case where $O$ is in III, we consider the responses at $N 2, N 3$, and $W$ as $z_{N 2}, z_{N 3}$, and $z_{w}$, respectively. Let us define the $d z_{N 3 W}, d z_{N 2 W}$, and $d z_{N 2 N 3}$ as before. For the case $C=0$, it can be shown that $d z_{N 3 W}>0$, which is a contradiction for $z_{N 3}<z_{W}$. Similarly, for $C<0$ and $C>0$, we have that $d z_{N 2 W}>0$ and $d z_{N 2 N 3}>0$ are contradictions for $z_{N 2}<z_{W}$ and $z_{N 2}<z_{N 3}$. The last case is where $O$ is in IV. We consider the responses at $N 2, N 3, N 4$, and $W$. Let us define $d z_{N 4 W}$ as before. For the case $C=0$, it can be shown that $d z_{N 2 N 3}>0$, which is a contradiction for $z_{N 2}<z_{N 3}$. Similarly, for $C<0$ and $C>0$, we have that $d z_{N 2 N 3}>0$ and $\mathrm{d} z_{N 4 W}>0$ are contradictions for $z_{N 2}<z_{N 3}$ and $z_{N 4}<z_{W}$.

3.3.2. Best at corner $(B C O)$ optimal regions. The case when $B$ is located at a corner is referred as a $B C O$ optimal region. In $B C O$, either $N 2$ or N3 can occur at the center. For N2 at center, there are three possible ORs based on the location of $B, N 3, N 4$, and $W$ (Figure 8 ).

In the case with $N 3$ at center, there are two possible ORs based on the location of $B, N 2, N 4$, and $W$ (Figure 9).

The proving strategy for $B C O s^{\prime} O R s$ is the same as $B C E$ and is thus excluded. Note that the implied optimal regions are identical to the ORs in Figures 8(a), 8(b), and 9(b); thus, there is no relaxation due to rectangular envelope.

\subsection{Parametric approach: model fitting strategy}

We use a parametric approach based on model fitting in addition to the ranking approach. This strategy not only allows us to increase the precision of $E O_{r}$ but also supports backtracking through $F S_{r}$ correction as explained in Section 3.2. Beginning with the completion of all first run experiments, this parametric approach is used after each experiment. In this approach, we fit $z=\left[\begin{array}{ll}a_{1} & a_{2}\end{array}\right]\left[\begin{array}{l}x \\ y\end{array}\right]+$ $\left[\begin{array}{ll}x & y\end{array}\right]\left[\begin{array}{ll}q_{1} & q_{2} \\ q_{2} & q_{3}\end{array}\right]\left[\begin{array}{l}x \\ y\end{array}\right]+c+\varepsilon_{1}$ model with $\varepsilon \sim N\left(0, \sigma^{2}\right)$, to the experimental data to analyze the underlying function of data and efficacy of conducted experiments. In fitting the quadratic model, two objectives are being sought in particular: (i) estimating the optimal experiment $E O_{r}$ and (ii) calculating the adjusted coefficient of determination $\left(R_{\text {adj }}^{2}\right) \cdot E O_{r}$, the minimum of the fitted model, not only can show the predicted optimal solution but can also be used for correcting the $F S_{r}$ of the next run. Furthermore, the change in the $E O_{r}$ in consecutive runs is also used as a stopping criterion. In comparison, the $R_{\text {adj }}^{2}$ shows how well the information gained from the experiments explains the behavior of the underlying system. ${ }^{32}$ We also use this measure as a stopping rule in the proposed ASRSM methodology and for comparing the explanatory power of different methods.

\subsection{Design structure for the next runs}

Following the characterization of $O R_{r}$ through the ranking approach and the estimation of the $E O_{r}$ from the parametric approach, we determine the design structure for the next run. In particular, we compare the $E O_{r}$ from the model fitting with the $O R_{r}$ from the

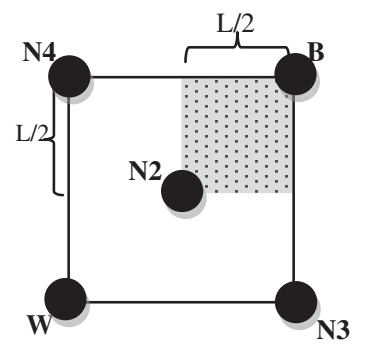

(a) Corner Design with B cornered with N3 and N4

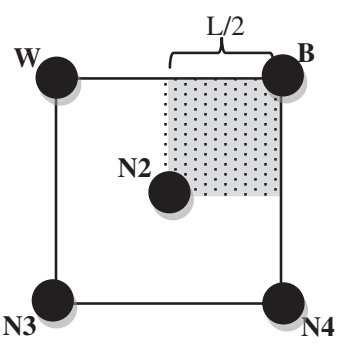

(b) Corner Design with B cornered with N4 and W

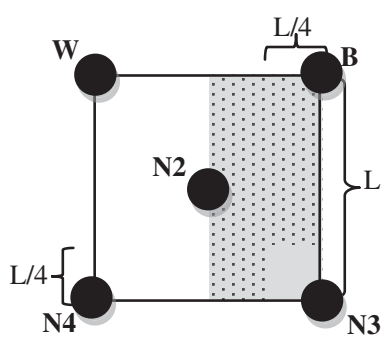

(c) Corner Design with B cornered with $\mathrm{N} 3$ and $\mathrm{W}$

Figure 8. $B C O$ ORs when $N 2$ is at the center (dotted region, implied $O R$; shaded region, $O R$ )

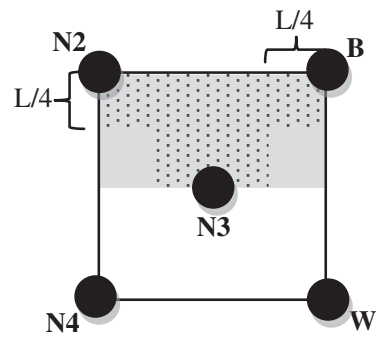

(a) Corner Design with B cornered with $\mathrm{N} 2$ and $\mathrm{W}$

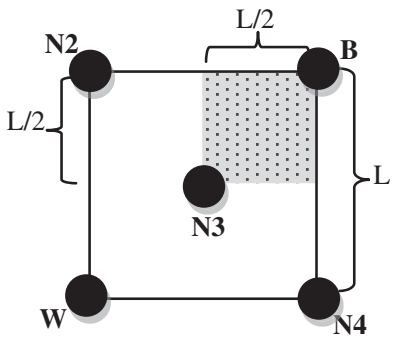

(b) Corner Design with B cornered with N2 and N4

Figure 9. $B C O$ ORs when $N 3$ is at the center (dotted region, implied $O R$; shaded region, $O R$ ) 
experiment ranking. If $E O_{r}$ is contained in the $O R_{r}$, then we use the region as the factor space of the next run. If $E O_{r}$ is not contained in the $O R_{r}$, we then expand the optimal region to a larger rectangle envelope containing the $E O_{r}$ and use the region as $F S_{r}$ of the next run (Figure 10). Next, we conduct experiments on the un-experimented corners and the center of the new $F S_{r}$. After each experiment, we fit the quadratic model and check whether $E O_{r}$ is contained in $O R_{r}$. If $E O_{r}$ is outside $O R_{r}$, then we expand the $O R_{r}$ as before. This expansion serves as a backtracking step. These steps are repeated to monitor the change in $R_{\text {adj }}^{2}$ and the $E O_{r}$ using the fitted model. The stopping condition for the proposed ASRSM approach is the convergence of $R_{\text {adj }}^{2}$ or $E O_{r}$ with thresholds $\delta_{\mathrm{EO}}$ and $\delta_{R_{\mathrm{adj}}^{2}}$.

\section{Applications of the adaptive sequential response surface methodology}

In this section, we first illustrate the application of the proposed ASRSM approach through a stylized example and compare its performance with the traditional CCD approach. Next, we experiment with the proposed ASRSM, CCD, and three optimal designs using the well-known paper helicopter experiment. Lastly, we report on the results of a rat brain trauma case study comparing ASRSM and CCD approaches.

\subsection{Illustrative example}

We consider the quadratic response model of the form $Z=X^{2}+2 Y^{2}-2 Y+\varepsilon$ with $\varepsilon \sim N\left(0,2^{2}\right)$, which is desired to be minimized. The starting region of interest is selected as $X \in[-3,3]$ and $Y \in[-3,3]$, and the contour plot of the response is presented in Figure 11 (a). We first conducted a typical CCD with 13 experiments centered at $(0,0)$ and contains the optimal experiment $O=(0,0.5)$ with mean response $Z_{O}=-0.5$ (Figure $11(\mathrm{a})$ ).

On the basis of the 13 experiments, the CCD attains $R_{\mathrm{adj}}^{2}=60.86 \%$ with $\left(X^{*}, Y^{*}\right)=(0.5,0.8428)$ as estimation of the optimal experiment. Figure 11 (b) illustrates the estimated contours using CCD design. The reason that CCD could not estimate the orientation of the quadratic response is the large magnitude of the variance of error term in the quadratic surface equation.

Next, we employ the ASRSM and present the results in Table I. The first five rows correspond to the initial run design. Note that the ASRSM is setup as described in Section 3.2 and without any additional information than used in the CCD. We use $R_{\text {adj }}^{2} \geq \theta_{R_{\text {adj }}^{2}}=85 \%$ as the convergence criteria in this example. We now describe each run in detail.

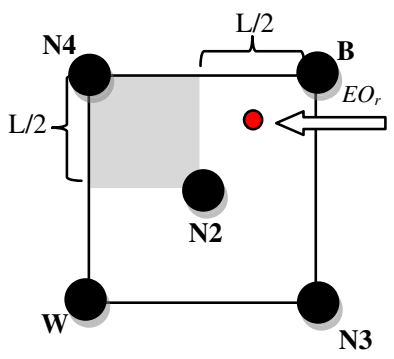

(a) Initial $F S_{r}$ not including $E O_{r}$

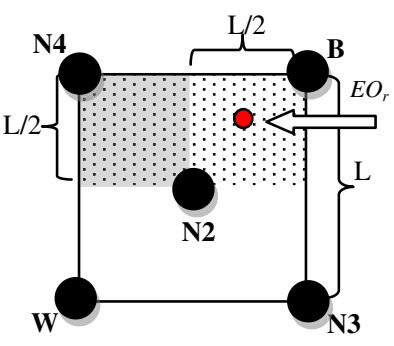

(b) Increased $F S_{r}$ based on

Figure 10. Expansion of the $O R_{r}$ when the $E O_{r}$ from model fitting falls outside

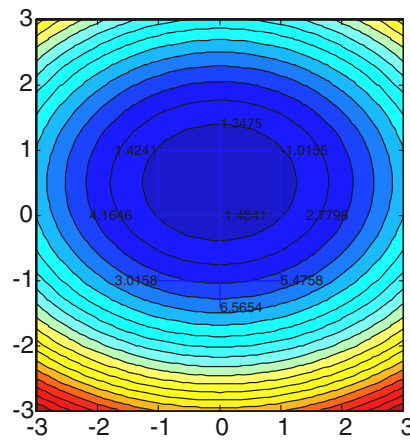

(a) Real contours

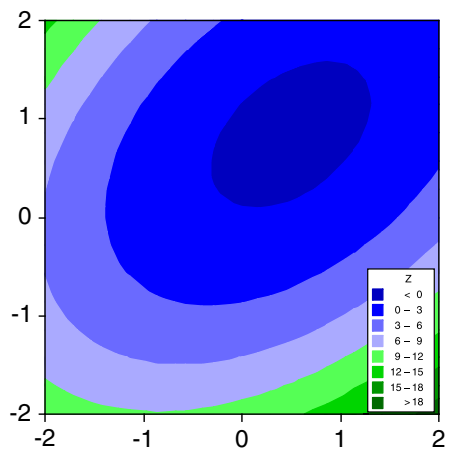

(b) CCD contours

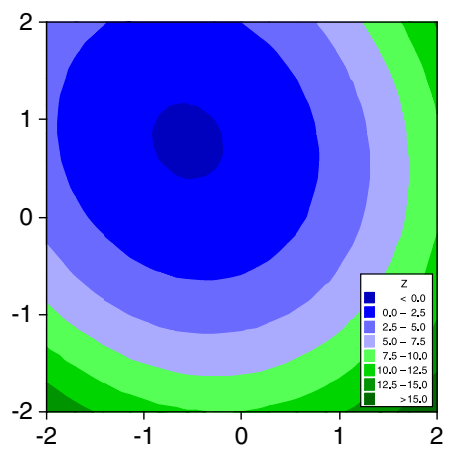

(c) ASRSM contours

Figure 11. The (a) actual, (b) central composite design, and (c) adaptive sequential response surface methodology estimated contours of the response $Z$ 


\begin{tabular}{|c|c|c|c|c|}
\hline \multirow{2}{*}{$\begin{array}{l}\text { Run } \\
\text { no. }\end{array}$} & \multirow[b]{2}{*}{ No. of new experiments } & \multicolumn{2}{|c|}{ Factor combination } & \multirow{2}{*}{$\frac{\text { Response }}{Z}$} \\
\hline & & $x$ & $Y$ & \\
\hline \multirow[t]{5}{*}{1} & 1 & -1.4142 & -1.4142 & 8.2950 \\
\hline & 2 & 1.4142 & -1.4142 & 10.2316 \\
\hline & 3 & -1.4142 & 1.4142 & 2.1963 \\
\hline & 4 & 1.4142 & 1.4142 & 6.8961 \\
\hline & 5 & 0 & 0 & 2.2137 \\
\hline \multirow[t]{3}{*}{2} & 6 & -1.4142 & 0 & -0.4552 \\
\hline & 7 & 1.4142 & 0 & -0.1682 \\
\hline & 8 & -0.7071 & 0.7071 & 0.8719 \\
\hline 3 & 9 & 0 & .3535 & 0.4117 \\
\hline
\end{tabular}

\section{Run 1}

Given the initial design, we obtain the $O R_{1}$ using the non-parametric approach (Figure 12(a)). The constrained quadratic fit estimates the optimal experiment $\left(E O_{1}\right)$ as $(-0.3572,1.4142)$, illustrated with a small point on the edge $B-N 3$ (Figure 12 (b)). Note that because the number of experiments is not sufficient to estimate the full model, the $E O_{1}$ is estimated by the quadratic response model without the constant term. Because the $E O_{1}$ is contained in the $O R_{1}$, the $O R_{1}$ is final. The new factor space $F S_{2}$ is determined as $O R_{1}$.

\section{Run 2}

Figure 12(c) shows the location of the first new experiment in $r=2$ that corresponds to $(-1.4142,0)$. Using the constrained quadratic fit as before, we estimate the optimal experiment as $(-1.4142,0.5930)$ illustrated with a small point (Figure 12(c)). Because this experiment is contained in $\mathrm{FS}_{2}$, there is no update of the factor space. The second new experiment is illustrated in Figure 12(d) and the corresponding estimate of the optimal experiment $(-0.7,0.6428)$ with $R_{\text {adj }}^{2}=51.96 \%$. This estimated optimum is still contained in $\mathrm{FS}_{2}$. The third and final experiment of $r=2$ is shown on Figure 12(e) together with the estimated optimal experiment $(-.5571,0.7857)$ and $R_{\text {adj }}^{2}=84.44 \%$.

The second run of the experiment terminates with the estimated optimal experiment in Figure 12(e). Continuing to the third run, the final $O R_{2}$ and $F S_{3}$ are illustrated in Figure 12(f). The first experiment in $r=3$ is the corner point of $F S_{3}$ indicated with dotted point. This point is the last experiment in Table I. The estimated optimal experiment with this experiment is $(0.5571,0.7851)$ with $R_{\mathrm{adj}}^{2}=89.06$, which satisfies the termination criteria. Figure 11(c) shows the estimated contours using the proposed approach. In this particular example, the response model estimation based on ASRSM is better than CCD.

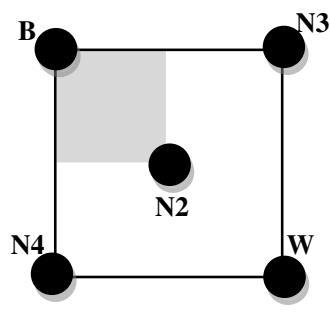

(a) Experiment Ranking $r=1$ and $O R_{l}$

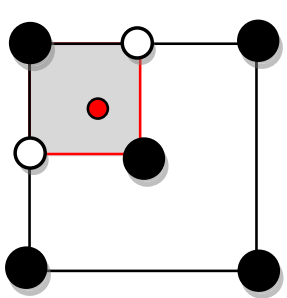

(d) 2nd new experiment $(r=2)$

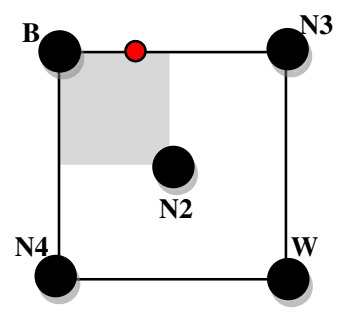

(b) $E O$ for $r=1$ and final $O R_{l}$

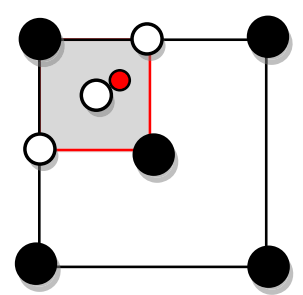

(e) 3rd new experiment $(r=2)$

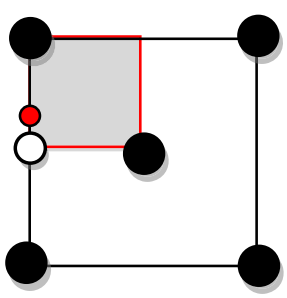

(c) 1st new experiment $(r=2)$

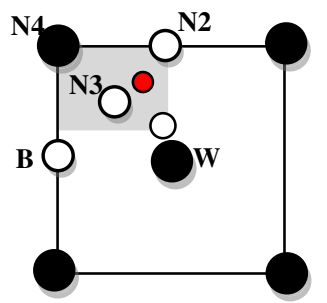

(f) Experiment ranking and $E O$ for $r=2$, and final $\mathrm{OR}_{2} \equiv F S_{3}$

Figure 12. Illustration of the steps of the adaptive sequential response surface methodology for runs 1 and 2 


\subsection{Paper helicopter}

Paper helicopter problem is a simple practical experiment that is frequently used for teaching as well as testing for different methods. Paper helicopter problem consists of studying the effect of a number of factors, that is, wing length, body length, and so on, on the flying time of a paper helicopter (Figure 13). Using this practical experiment, we now compare the performances of ASRSM, CCD, A-optimal, D-optimal, and V-optimal designs.

The paper helicopter problem is originally designed for the design of experiments. In order to apply alternative approaches to this problem, we first extended it to the RSM domain. For this, we first chose the wing length and the body length as the two controllable factors under study. Next, we conducted a number of experiments to find an initial feasible range for each of the designated variables: wing length $\left(4.50 \mathrm{~cm}<X_{1}<10.25 \mathrm{~cm}\right)$ and body length $\left(3.75 \mathrm{~cm}<X_{2}<9.25 \mathrm{~cm}\right)$ that contains the optimal region. Finally, we have applied the ASRSM, CCD, A-optimal, D-optimal, and V-optimal designs to the coded factor space and compared their performances. Note that we are reporting on the CCD results with 9 and 13 experiments, where the latter has four additional center experiments. All other approaches are based on eight experiments. In total, we have conducted 53 experiments. Table II present the coded design points and the corresponding responses for each method.

Table III presents the prediction results of the paper helicopter experiment. Because the true response model is unknown, we chose to compare the methods using the estimated optimality gap, which is based on the best estimate of the optimum obtained by a response surface fit to all 53 experiments. On the basis of this estimated response, the optimal experiment is identified at $O=(1.0714,-0.007)$ with mean response $Z_{O}=2.59487 \mathrm{~s}$. Both the $R_{\text {adj }}^{2}$ and optimality gap results show that the ASRSM method outperforms other preset and optimal designs. The D-optimal and V-optimal designs are the second and third best performing approaches, respectively. Whereas the differences in the optimality gap are small, the differences in the Euclidean distance between the predicted optimal experiment $\hat{X}_{O}$ and optimal experiment $O$, for example, $\left\|O-\hat{X}_{0}\right\|$, are more substantial. This is attributable to the fact that quadratic convex functions are flat around the optimum and that the response is relatively insensitive to deviations from the optimum.

On the basis of the 13 experiments, CCD achieves $R_{\text {adj }}^{2}=50.81 \%$ with the estimated coded optimal solution $(0.957094,-0.242845)$, and the contours of the estimated fly time response model is shown in Figure 14(a). On the other hand, the ASRSM required only eight experiments in two runs to attain $R_{\mathrm{adj}}^{2}=86.54$ with the estimated coded optimal $(1.0427,0.0429)$. The contour plot of the estimated fly time response model is shown in Figure 14(b). These results clearly show that the ASRSM outperforms the traditional RSM CCD method in terms of both the number of experiments and the accuracy of the results.

\subsection{Traumatic brain injury: design of controlled cortical impact model}

Traumatic brain injury continues to be a serious societal problem that affects more than 1.4 million Americans each year. ${ }^{33}$ The controlled cortical impact $(\mathrm{CCl})$ rat model is one of the most frequently used animal models. This model is used to correlate real-world injuries with predictions from a validated finite element model in order to establish injury threshold. In CCI model, the impact depth (potentially ranging from 1 to $3 \mathrm{~mm}$ ) and the impactor diameter (potentially ranging from 2.5 to $7.5 \mathrm{~mm}$ ) are believed to be the two main factors in determining injury severity. However, the percent of increase/decrease in the size of rat brain contributes to variances observed in post-impact tissues. Because the effect of this external parameter is largely unknown, it can be considered as noise. In CCI

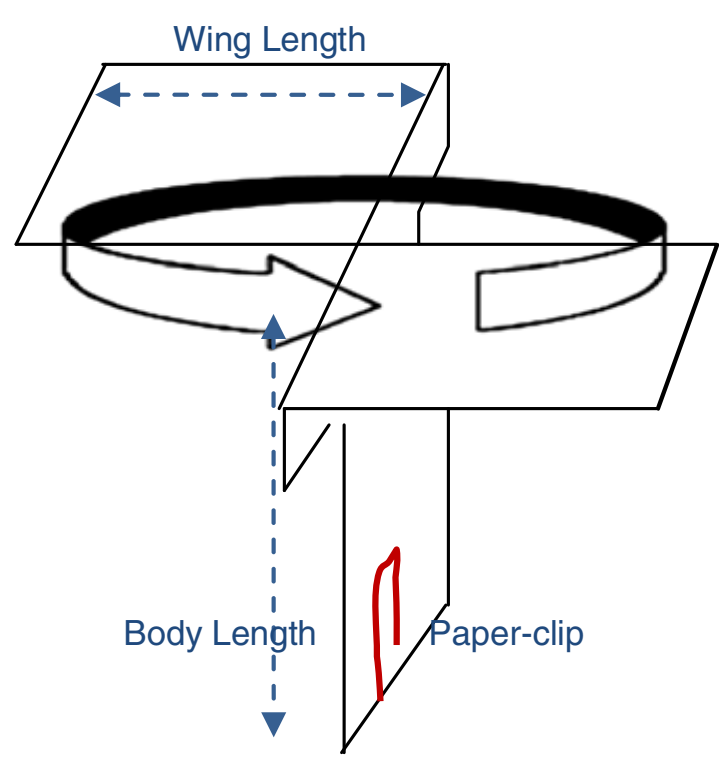

(a) Completed design

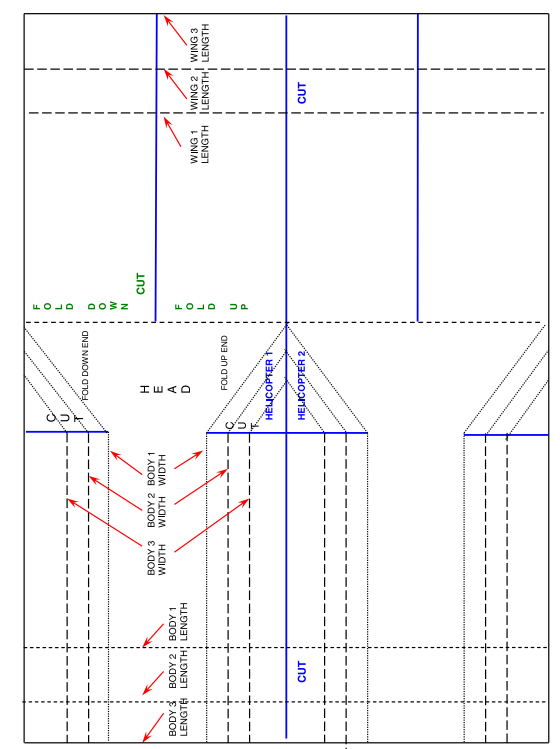

(b) Design template

Figure 13. Paper helicopter 
Table II. The runs and experiments of the adaptive sequential response surface methodology (ASRSM), central composite design (CCD), A-optimal, D-optimal, and V-optimal designs for the paper helicopter experiment

\begin{tabular}{|c|c|c|c|c|c|c|c|c|}
\hline \multirow[b]{2}{*}{ Exp. no. } & \multicolumn{3}{|c|}{ ASRSM } & \multicolumn{3}{|c|}{ CCD-9 } & \multicolumn{2}{|c|}{ CCD-13 } \\
\hline & $X_{1}^{\prime}$ & $x_{2}^{\prime}$ & Fly time (s) & $x_{1}^{\prime}$ & $x_{2}^{\prime}$ & Fly time (s) & $x_{1}^{\prime}$ & $x_{2}^{\prime}$ \\
\hline 1 & -1.414 & -1.414 & 1.47 & -1 & -1 & 1.85 & -1 & -1 \\
\hline 2 & 1.414 & -1.414 & 2.34 & 1 & -1 & 2.19 & 1 & -1 \\
\hline 3 & -1.414 & 1.414 & 1.53 & -1 & 1 & 1.75 & -1 & 1 \\
\hline 4 & 1.414 & 1.414 & 2.50 & 1 & 1 & 2.22 & 1 & 1 \\
\hline 5 & 0 & 0 & 2.46 & -1.414 & 0 & 1.59 & -1.414 & 0 \\
\hline 6 & 0 & 1 & 2.13 & 1.414 & 0 & 2.6 & 1.414 & 0 \\
\hline 7 & 0 & -1.414 & 2.41 & 0 & -1.414 & 2.41 & 0 & -1.414 \\
\hline 8 & 1 & 0 & 2.57 & 0 & 1.414 & 2.13 & 0 & 1.414 \\
\hline 9 & & & & 0 & 0 & 2.5 & 0 & 0 \\
\hline 10 & & & & & & & 0 & 0 \\
\hline 11 & & & & & & & 0 & 0 \\
\hline 12 & & & & & & & 0 & 0 \\
\hline 13 & & & & & & & 0 & 0 \\
\hline
\end{tabular}

$X_{1}^{\prime}$ and $X_{2}^{\prime}$ correspond to the coded wing length and body length factors, respectively.

studies, one common problem is to find the specific levels of factors that result in specific percent of injury in animal brain. However, these experiments are not only very expensive but also very time consuming.

In this case study, we used the proposed approach to find the parameter setting that results in $30 \%$ injury in the rat brain. We also conducted CCD experiments to compare the performance with the proposed approach. The technical details of the experiments can be found in Mao et al. ${ }^{33}$ Table IV shows the conducted experiments of CCD and the proposed ASRSM approach at different runs.

Using 13 experiments, the CCD fits a quadratic surface with $R_{\mathrm{adj}}^{2}=68.31 \%$ and identifies $E O=(0.1857,0.3286)$. Figure 15 (a) shows the 3D plot of CCD estimated surface. In comparison, with the use of eight experiments in two runs, the proposed approach fits the quadratic model shown in Figure $15(\mathrm{~b})$ with $R_{\mathrm{adj}}^{2}=82.59 \%$ and $E O=(0,0.0505)$. As shown in Figure 15 , although the estimated optimal experiments of both approaches are close, the estimated response models of the two methods are significantly different. The differences are even more apparent from the contours of the two response model estimates (Figure 16).

To compare the estimated functions, we aggregate the experimental data from both approaches (e.g., Table IV) and used the radial basis function (RBF) to find the best fit. Figure 15(c) illustrates the model fit using RBF and the aggregated experimental data. From Figure 15, it can be seen that the estimated function of the proposed approach is much more similar to RBF with aggregated experimental data. Meanwhile, we also note that the $E O$ of the proposed approach is very close to that of RBF based on the contour plots in Figure 15(b), (c). Given that the underlying response model is potentially highly nonlinear, the results are very encouraging for the effectiveness of the proposed approach in real-world applications.

\section{Performance comparison of ASRSM}

We now describe the simulated experiments performed to compare the performance of the proposed ASRSM approach with those of CCD, A-optimal, D-optimal, and V-optimal designs. In the simulated experiments, we considered six response models with varying variance of error and function type (i.e., convex and non-convex). These response models are presented in Table V. All response models have a quadratic relation, for example, $=A X^{2}+B Y^{2}+C X+D Y+E X Y+F+\varepsilon$, with a normal error term $\varepsilon \sim N\left(0, \sigma^{2}\right)$.

Whereas the ASRSM is an adaptive sequential method, the CCD, A-optimal, D-optimal, and V-optimal designs are essentially preset designs. In order to understand the effect of this difference, we have carried out two sets of analyses. In the first set, we have fixed the number of observations for each approach and compared the performances in terms of average $R_{\text {adj }}^{2}$ and average optimality gap (i.e., deviation from the optimal response). Given the optimal response $\left(Z_{O}\right)$, the optimality gap of a method is defined as $\left(\hat{Z}-Z_{O}\right) / Z_{O}$, where $\hat{Z}$ is the mean predicted response at the estimated optimal point. All simulated experiments are repeated five times, and average results are reported. For each response model in Table V, the design points in A-optimal, D-optimal, and V-optimal designs are generated by optimizing the optimality criteria over the starting factor space with a fine grid system spaced with 0.01 intervals. The CCD design consists of four corner, four axial, and one center design points as in the illustrative example in Section 4.1. Note that the starting factor space expands the initial region of interest, $X \in[-3,3]$ and $Y \in[-3,3]$, by a factor of $\sqrt{2}$ in all directions as explained in Section 3.2 and illustrated in Figure 5(a).

Table VI presents the results of the first set of analyses. The ASRSM has a better $R_{\text {adj }}^{2}$ in four out of the six response models and has $R_{\text {adj }}^{2}=94.74 \%$ on the average. The A-optimal, D-optimal, and V-optimal designs are also competitive, and CCD has the worst average performance. With respect to the optimality gap, the proposed ASRSM has the best performance in all but one of the response models with an average gap of $16.02 \%$. This gap improved drastically by running the ASRSM for additional iterations, which is not reported here. The results indicate that increasing the variance of the response decreases the $R_{\text {adj }}^{2}$ for all approaches, for example, 


\begin{tabular}{|c|c|c|c|c|c|c|c|c|c|}
\hline CCD-13 & \multicolumn{3}{|c|}{ A-Optimal } & \multicolumn{3}{|c|}{ D-Optimal } & \multicolumn{3}{|c|}{ V-Optimal } \\
\hline Fly time (s) & $X_{1}^{\prime}$ & $x_{2}^{\prime}$ & Fly time (s) & $X_{1}^{\prime}$ & $X_{2}^{\prime}$ & Fly time (s) & $X_{1}^{\prime}$ & $x_{2}^{\prime}$ & Fly time (s) \\
\hline 1.85 & 1.414 & 1.414 & 2.41 & 1.414 & 1.414 & 2.41 & 1.414 & 1.414 & 2.41 \\
\hline 2.19 & -1.414 & -1.414 & 1.50 & -1.414 & -1.414 & 1.5 & 0.141 & 0.283 & 2.06 \\
\hline 1.75 & -1.414 & 1.414 & 1.54 & -1.414 & -0.141 & 1.47 & 1.414 & -0.990 & 2.75 \\
\hline 2.22 & 1.414 & -1.414 & 2.60 & 0 & -1.414 & 2.22 & -1.414 & -1.414 & 1.50 \\
\hline 1.59 & 0.283 & 0 & 2.15 & 0 & 0.141 & 2.32 & -1.414 & -0.141 & 1.47 \\
\hline 2.60 & -0.141 & 1.414 & 1.91 & -1.414 & 1.414 & 1.54 & 0 & -1.414 & 2.22 \\
\hline 2.41 & -1.414 & 0.000 & 1.60 & 1.414 & -1.414 & 2.6 & 0 & 0.141 & 2.32 \\
\hline 2.13 & -0.141 & -1.414 & 2.10 & 1.414 & -0.141 & 2.63 & -1.273 & 1.414 & 1.34 \\
\hline \multicolumn{10}{|l|}{2.50} \\
\hline \multicolumn{10}{|l|}{2.28} \\
\hline \multicolumn{10}{|l|}{2.25} \\
\hline \multicolumn{10}{|l|}{1.94} \\
\hline 2.46 & & & & & & & & & \\
\hline
\end{tabular}

Table III. Prediction results of the adaptive sequential response surface methodology (ASRSM), central composite design (CCD), and optimal designs for the paper helicopter case study

\begin{tabular}{|lllrrrrr} 
& & ASRSM & CCD-9 & CCD-13 & A-Optimal & D-Optimal & V-Optimal \\
\hline Adjusted $R^{2}(\%)$ & & 86.5 & 55.2 & 50.8 & 62.1 & 66.3 & 70.8 \\
Predicted optimum & $X_{1}$ & 1.0427 & 0.8624 & 0.9571 & 1.1142 & 1.1635 & 1.2856 \\
& $X_{2}$ & 0.0429 & -0.1021 & -0.2428 & -0.3842 & -0.4124 & -0.5451 \\
$\left\|O-\hat{X}_{0}\right\|$ & 0.0576 & 0.2296 & 0.2620 & 0.3796 & 0.4158 & 0.5792 & 2.5917 \\
Pred. resp. & $\hat{Z}$ & 2.5931 & 2.5783 & 2.5842 & 2.5904 & 2.5902 \\
Opt. gap & & $0.07 \%$ & $0.64 \%$ & $0.41 \%$ & $0.17 \%$ & $0.12 \%$ & $0.18 \%$ \\
\hline
\end{tabular}

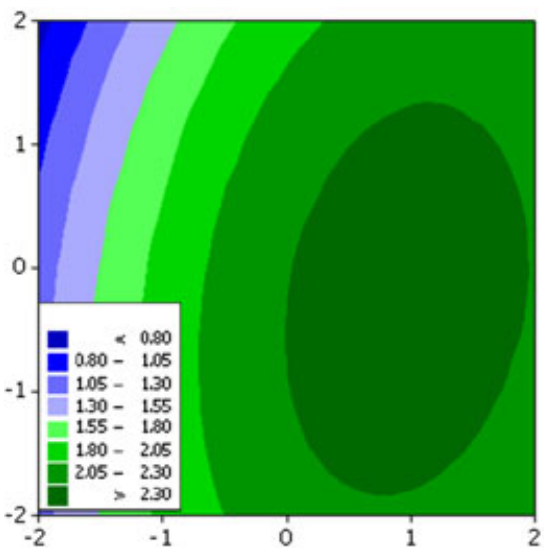

(a)

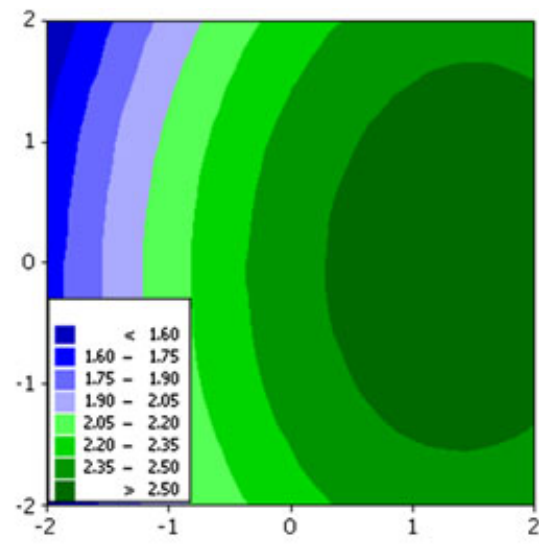

(b)

Figure 14. The (a) central composite design and (b) adaptive sequential response surface methodology estimated contours of the paper helicopter fly time response

response models 1 versus 3 and models 2 versus 6. However, this reduction is least with ASRSM. In the case of optimality gap, the increase in the variance of response increases (decreases) the optimality gap in convex (non-convex) response models. While the generalization of these effects requires further analysis, we note that the increase (decrease) in the optimality gap is least (most) with the proposed ASRSM. These results show that the ASRSM is competitive with the three optimal designs and outperforms the classical CCD design. In addition, the performance of ASRSM is more robust with respect to changes in the error variance and convexity of the response model. 


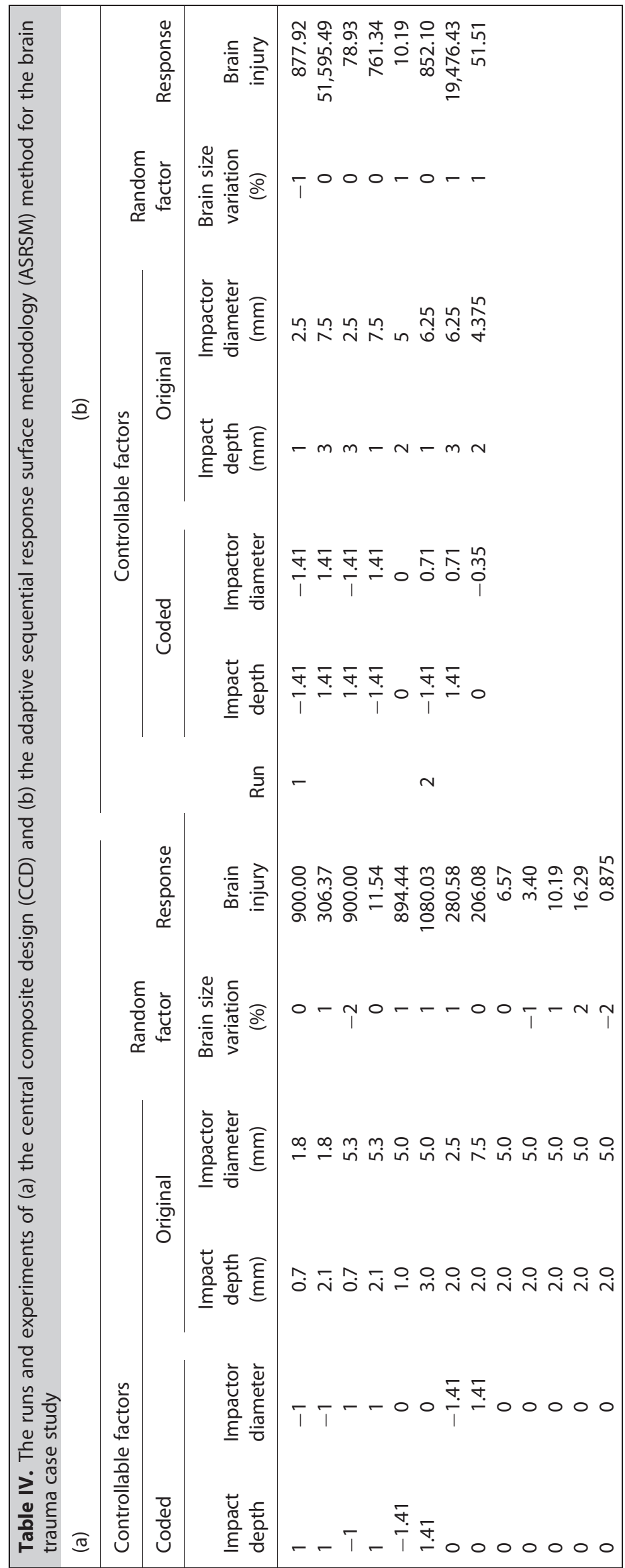




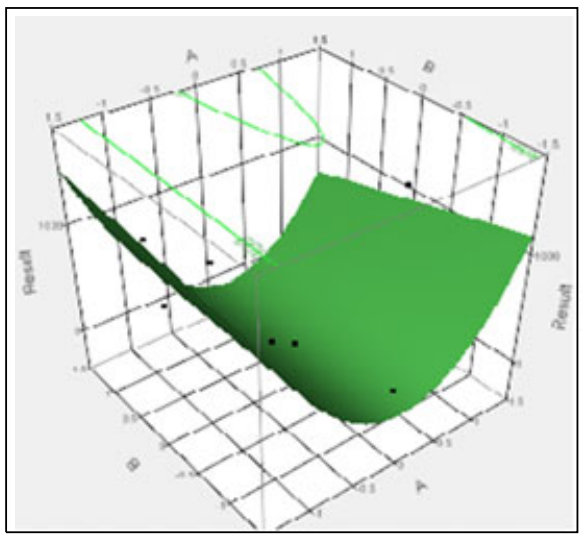

(a)

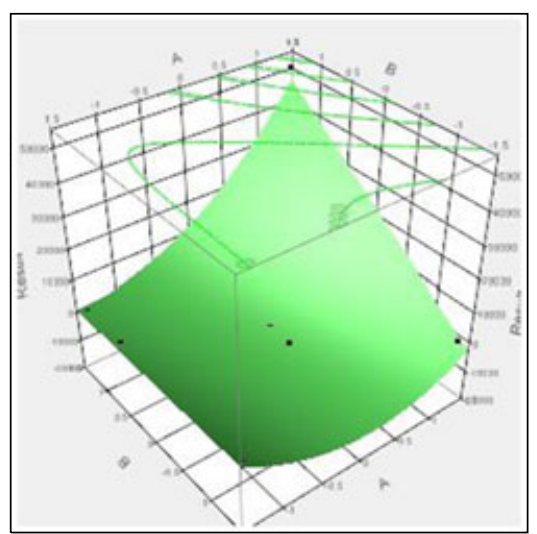

(b)

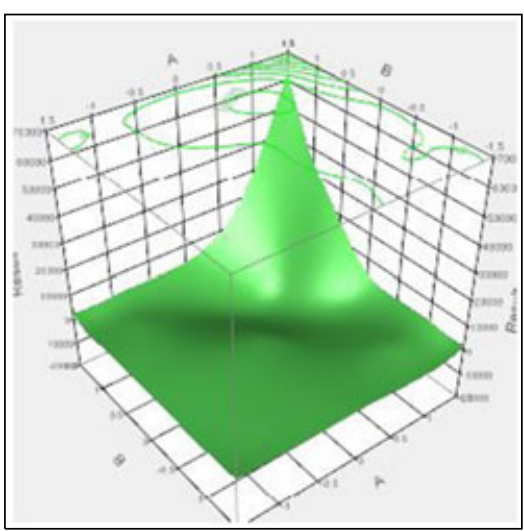

(c)

Figure 15. Three-dimensional plots of the estimated response for the (a) central composite design, (b) adaptive sequential response surface methodology, and (c) radial basis function in the brain trauma case study
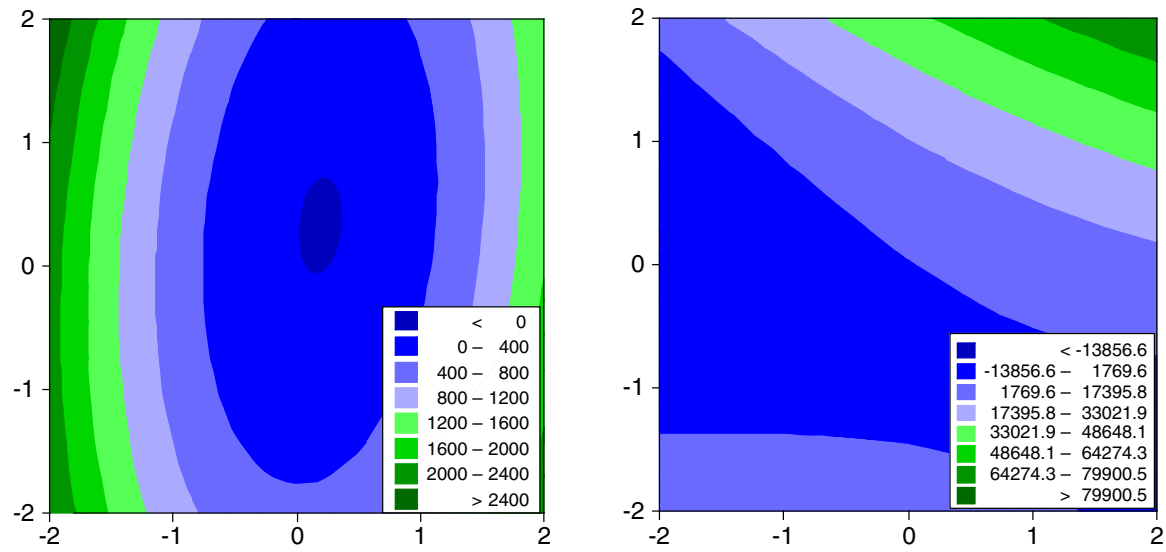

Figure 16. The (a) central composite design and (b) adaptive sequential response surface methodology estimated contours of the response in the brain trauma case study

\begin{tabular}{|c|c|c|c|c|c|}
\hline \multirow[b]{2}{*}{ Exp. no. } & \multirow[b]{2}{*}{ Response relation } & \multirow[b]{2}{*}{ Error $(\varepsilon)$} & \multirow{2}{*}{$\begin{array}{l}\text { Response } \\
\text { type }\end{array}$} & \multicolumn{2}{|c|}{ Optimal experiment } \\
\hline & & & & $O=\left(X_{\mathrm{O}}, Y_{\mathrm{O}}\right)$ & $Z_{\mathrm{O}}$ \\
\hline 1 & $Z=-2 x^{2}+3 y^{2}+2 x-y+2 x y-1+\varepsilon$ & $N(0,0.1)$ & Non-convex & $(-3.0,1.25)$ & -29.063 \\
\hline 2 & $Z=x^{2}+2 y^{2}-2 y+\varepsilon$ & $N(0,1)$ & Convex & $(0.0,0.5)$ & -0.500 \\
\hline 3 & $Z=-2 x^{2}+3 y^{2}+2 x-y+2 x y-1+\varepsilon$ & $N(0,2)$ & Non-convex & $(-3.0,1.25)$ & -29.063 \\
\hline 4 & $Z=-3 x^{2}+2 y^{2}+x-2 y+2 x y-1+\varepsilon$ & $N(0,2)$ & Non-convex & $(-3.0,2.0)$ & -39.000 \\
\hline 5 & $Z=2 x^{2}+y^{2}+x+2 x y+\varepsilon$ & $N(0,2)$ & Convex & $(0.5,-0.5)$ & 0.750 \\
\hline 6 & $Z=x^{2}+2 y^{2}-2 y+\varepsilon$ & $N(0,2)$ & Convex & $(0.0,0.5)$ & -0.500 \\
\hline
\end{tabular}

According to Table VI, the ASRSM's average performance improvement over other methods is more significant in optimality gap than in $R_{\text {adj. }}^{2}$ This is because the ASRSM searches for the optimal design point by sequentially contracting the factor space, whereas other approaches select the design points using the initial factor space. Hence, the design points used in ASRSM are more densely distributed than other methods. In order to capture this difference, we have carried another set of analyses for the optimal designs. In this second set, we initially fixed the number of design points at seven and then incrementally added one design point at a time until we have a total of nine design points. The initial set of seven design points is optimally generated as before. Next, each of the additional point is generated by optimizing the optimality criterion given the existing design points and the response model. At each run, we have compared the performances in terms of average $R_{\text {adj }}^{2}$ and average optimality gap. Note that we have used the earlier 
Table VI. $R_{\text {adj }}^{2}$ and optimality gap results of simulated experiments with nine observations determined $a$ priori for central composite design and optimal designs

\begin{tabular}{|c|c|c|c|c|c|c|c|c|c|c|}
\hline \multirow[b]{2}{*}{ Exp. no. } & \multicolumn{5}{|c|}{ Adjusted $R^{2}(\%)$} & \multicolumn{5}{|c|}{ Optimality gap (\%) } \\
\hline & ASRSM & $C C D$ & A-Optimal & D-Optimal & V-Optimal & ASRSM & $C C D$ & A-Optimal & D-Optimal & V-Optimal \\
\hline 1 & 99.98 & 99.94 & 99.89 & 99.98 & 99.96 & 10.43 & 105.77 & 15.10 & 72.35 & 97.67 \\
\hline 2 & 97.38 & 91.78 & 94.08 & 95.63 & 97.77 & 17.17 & 55.53 & 15.59 & 18.16 & 19.21 \\
\hline 3 & 93.36 & 78.56 & 88.43 & 93.61 & 85.55 & 7.34 & 87.23 & 9.25 & 9.50 & 68.93 \\
\hline 4 & 94.38 & 84.74 & 91.27 & 90.18 & 84.43 & 13.85 & 58.06 & 14.01 & 18.47 & 17.84 \\
\hline 5 & 94.28 & 37.83 & 87.70 & 80.69 & 88.04 & 29.33 & 684.08 & 78.93 & 70.68 & 144.60 \\
\hline 6 & 89.06 & 49.32 & 78.31 & 81.72 & 86.57 & 18.02 & 179.17 & 354.21 & 85.41 & 71.45 \\
\hline Ave. & 94.74 & 73.69 & 89.95 & 90.30 & 90.39 & 16.02 & 194.97 & 81.18 & 45.76 & 69.95 \\
\hline
\end{tabular}

ASRSM, adaptive sequential response surface methodology; CCD, central composite design.

\begin{tabular}{|c|c|c|c|c|c|c|}
\hline \multirow[b]{2}{*}{ Exp. no. } & \multirow[b]{2}{*}{ No of obs. } & \multicolumn{5}{|c|}{ Adjusted $R^{2}(\%)$} \\
\hline & & ASRSM & $C C D$ & A-Optimal & D-Optimal & V-Optimal \\
\hline \multirow[t]{3}{*}{1} & 7 & 99.88 & 99.82 & 99.79 & 99.80 & 99.91 \\
\hline & 8 & 99.89 & 99.73 & 99.91 & 99.86 & 99.89 \\
\hline & 9 & 99.98 & 99.94 & 99.88 & 99.96 & 99.91 \\
\hline \multirow[t]{3}{*}{2} & 7 & 94.83 & 92.08 & 91.12 & 94.18 & 93.92 \\
\hline & 8 & 95.65 & 92.08 & 87.51 & 89.49 & 94.99 \\
\hline & 9 & 97.38 & 91.78 & 86.79 & 90.11 & 94.89 \\
\hline \multirow[t]{3}{*}{3} & 7 & 80.04 & 72.09 & 97.51 & 92.78 & 71.98 \\
\hline & 8 & 86.99 & 73.07 & 79.53 & 90.66 & 75.00 \\
\hline & 9 & 93.36 & 78.56 & 82.11 & 89.04 & 80.93 \\
\hline \multirow[t]{3}{*}{4} & 7 & 91.75 & 92.75 & 94.23 & 90.84 & 84.85 \\
\hline & 8 & 92.51 & 88.11 & 89.25 & 91.70 & 88.21 \\
\hline & 9 & 94.38 & 84.74 & 89.82 & 92.69 & 85.59 \\
\hline \multirow[t]{3}{*}{5} & 7 & 90.17 & 24.90 & 87.32 & 87.22 & 86.03 \\
\hline & 8 & 89.05 & 36.02 & 89.67 & 76.75 & 72.07 \\
\hline & 9 & 94.28 & 37.83 & 86.64 & 68.07 & 85.07 \\
\hline \multirow[t]{3}{*}{6} & 7 & 86.09 & 35.34 & 50.18 & 85.09 & 84.97 \\
\hline & 8 & 86.78 & 43.18 & 56.96 & 78.61 & 86.13 \\
\hline & 9 & 89.06 & 49.32 & 64.13 & 68.54 & 81.92 \\
\hline \multirow[t]{3}{*}{ Ave. } & 7 & 90.46 & 69.50 & 86.69 & 91.65 & 86.92 \\
\hline & 8 & 91.81 & 72.03 & 83.80 & 87.84 & 86.05 \\
\hline & 9 & 94.74 & 73.69 & 84.90 & 84.73 & 88.05 \\
\hline
\end{tabular}

ASRSM, adaptive sequential response surface methodology; CCD, central composite design.

\begin{tabular}{|c|c|c|c|c|c|}
\hline \multirow[b]{2}{*}{ Exp. no. } & \multicolumn{5}{|c|}{ Optimality gap (\%) } \\
\hline & ASRSM & $C C D$ & A-Optimal & D-Optimal & V-Optimal \\
\hline 1 & 10.43 & 105.77 & 22.44 & 78.54 & 123.69 \\
\hline 2 & 17.17 & 55.53 & 14.93 & 16.62 & 38.11 \\
\hline 3 & 7.34 & 87.23 & 10.25 & 11.10 & 191.25 \\
\hline 4 & 13.85 & 58.06 & 14.11 & 20.67 & 23.24 \\
\hline 5 & 29.33 & 684.08 & 86.76 & 75.27 & 405.52 \\
\hline 6 & 18.02 & 179.17 & 421.10 & 99.62 & 91.40 \\
\hline Ave. & 16.02 & 194.97 & 94.93 & 50.30 & 145.54 \\
\hline
\end{tabular}

ASRSM, adaptive sequential response surface methodology; CCD, central composite design. 
results of the ASRSM for consistency. For the CCD, we initially used seven of the earlier observations by excluding two axial points and then included them back one at a time.

The results of the $R_{\mathrm{adj}}^{2}$ for the consecutive runs are presented in Table VII. For ASRSM and CCD, the inclusion of additional design points increases the average $R_{\text {adj }}^{2}$ across all response models. In comparison, the average $R_{\text {adj }}^{2}$ decreases with additional design points for the A-optimal and D-optimal designs. Tables VI and VII show that applying A-optimal and D-optimal designs sequentially reduces the average $R_{\text {adj }}^{2}$. The performance of the V-optimal design is observed to increase. Table VIII presents the optimality gap results of the final nine design points in Table VII. Tables VI and VIII show that the optimality gap of A-optimal and D-optimal designs slightly deteriorated when applied in sequence. In contrast, the optimality gap of V-optimal design has dramatically worsened when applied in sequence. The results in Tables VII and VIII also show that the ASRSM method exhibits monotonic behavior when the number of observations is increased and is thus more suitable for sequential experimentation.

\section{Conclusions}

An adaptive methodology for response surface optimization (ASRSM), combining concepts from nonlinear optimization, design of experiments, and response surface optimization, is proposed. This sequential adaptive experimentation approach simultaneously reduces the region of interest and identifies factor combinations for new experiments by using the information gained from the previous experiments. Results from the extensive simulated experiments and real-world case studies show that the proposed method outperforms the CCD method and is competitive with the A-optimal, D-optimal, and V-optimal designs in terms of both optimality as well as experimentation efficiency. In particular, the performance of ASRSM is found to be more robust with respect to changes in the error variance and convexity of the response model and more monotonic with additional experiments. In the engineering design, computation-intensive design analyses are commonly expensive computer 'experiments' and thus require experimental optimization for design optimization. ${ }^{28-30}$ While the proposed approach targets industrial experiments with high experimentation cost, it can also be applied to computer experiments applied to engineering design optimization problems in two dimensions with convex quadratic objective functions.

There are three limitations for the proposed approach. First limitation is that the method is primarily developed for two significant and controllable factors. For instances with more than two factors, it is possible to sequentially apply the proposed method by keeping all other factors but two fixed and then sequentially choosing another pair of factors. Note that this is indeed an extension of the OFAT to two factors at a time, where the proposed method can be used in each iteration. The integration of the proposed method with the OFAT and determining the best strategy to select two factors at-a-time is the focus of future research. Another limitation is the quadratic convex relationship between the response and the two factors. Further extension of the proposed methodology will consider extending the ideas presented to higher order of response functions that are non-convex. One of the key principles of good design strategy is to randomize the sequence of trials of an experiment to eliminate the effects of unknown or uncontrolled factors. The last limitation of the ASRSM approach is the inability to achieve complete randomization because of its sequential characteristic. In other words, while the new trials can be randomized within each run, the ASRSM cannot achieve complete randomization across runs.

\section{Acknowledgement}

We are grateful to the anonymous reviewer whose valuable suggestions and comments improved the technical content and presentation clarity of this manuscript.

\section{References}

1. Spendley GR, Hex GR, Himsworth FR. Sequential application of simplex designs in optimization and evolutionary operation. Technometrics 1962; 4:441-461.

2. Gramacy RB, Lee HKH. Adaptive design and analysis of supercomputer experiments. Technometrics 2009; 51(2):130-145.

3. Gu L. A comparison of polynomial based regression models in vehicle safety analysis. In American Society of Mechanical Engineers Design Engineering Technical Conferences_Design Automation Conference, Diaz A (ed). American Society of Mechanical Engineers: New York, 2001, Paper No. DETC2001/DAC-21063.

4. Box GEP. Statistics as a catalyst to learning by scientific method: part II—a discussion. Journal of Quality Technology 1999; 31(1):16-29.

5. Myers RH. Response surface methodology_current status and future directions. Journal of Quality Technology 1999; 31:30-44.

6. Box GEP, Wilson KB. On the experimental attainment of optimum conditions. Journal of the Royal Statistical Society 1951; 13:1-15.

7. Friedman M, Savage LJ. Planning experiments seeking maxima. In Techniques of Statistical Analysis, Eisenhart C, Hastay M, Wallis WA (eds). McGraw-Hill: NY, 1947; 363-372.

8. Daniel C. One-at-a-time plans. Journal of the American Statistical Association 1973; 68:353-360.

9. Frey DD, Engelhardt F, Greitzer EM. A Role for one factor at a time experimentation in parameter design. Research in Engineering Design 2003; 14:65-74.

10. Wang G, Dong Z, Aitchison P. Adaptive response surface method-a global optimization scheme for computation-intensive design problems. Journal of Engineering and Optimization 2001; 33(6):707-734.

11. Wang G. Adaptive response surface method using inherited Latin hypercube designs. ASME Journal of Mechanical Design 2003; 125(2):210-220. 
12. Stander N. The Successive response surface method applied to sheet-metal forming. In Proceedings of the First MIT Conference on Computational Fluid and Solid Mechanics, Boston, June 12-14, 2001; Elsevier Science Ltd: Oxford.

13. Box GEP, Draper NR. Evolutionary Operation: A Method for Increasing Industrial Productivity. John Wiley and Sons: NY, 1969.

14. Hettmansperger TP, McKean JW. Robust Nonparametric Statistical Methods. Arnold/Wiley: London and New York, N.Y., 1998.

15. Myers RH, Khuri Al, Carter WH. Response surface methodology: 1966-1988. Technometrics 1989; 31:137-157.

16. Myers RH, Montgomery DC, Vining CG, Borror CM, Kowalski SM. Response surface methodology: a retrospective and literature survey. Journal of Quality Technology 2004; 36:53-77.

17. Box GEP, Behnken DW. Some new three level design for the study of quantitative variables. Technometrics 1960; 2:455-476.

18. Montgomery DC. Design and Analysis of Experiments (7th edn). John Wiley \& Sons: NJ, 2008.

19. Kiefer J. Optimum experimental designs. Journal of the Royal Statistical Society B 1959; 21:272-304.

20. Kiefer J. Optimum designs in regression problems. Annals of Mathematical Statistics 1961; 32:298-325.

21. Kiefer J, Wolfowitz J. Optimum designs in regression problems. Annals of Mathematical Statistics 1959; 30:271-294.

22. Pukelsheim F. Optimal Design of Experiments. Society for Industrial and Applied Mathematics, (SIAM): Philadelphia, PA, 2006.

23. Box GEP. Evolutionary operation: a method for increasing industrial productivity. Applied Statistics 1957; 6:81-101.

24. Box GEP, Hunter WG, Hunter JS. Statistics for Experimenters: An Introduction to Design, Data Analysis, and Model Building. John Wiley and Sons: NY, 1978.

25. Czitrom V. One-factor-at-a-time versus designed experiments. The American Statistician 1999; 53(2):126-131.

26. Frey DD, Jugulum R. The mechanisms by which adaptive one-factor-at-a-time experimentation leads to improvement. ASME Journal of Mechanical Design 2006; 128:1050-1060.

27. Frey DD, Wang H. Adaptive one-factor-at-a-time experimentation and expected value of improvement. Technometrics 2006; 48(3):418-431.

28. Jones DR. A taxonomy of global optimization methods based on response surfaces. Journal of Global Optimization 2001; 21(4):345-383.

29. Sobieszczanski-Sobieski J, Haftka RT. Multidisciplinary aerospace design optimization: survey of recent developments. Structural and Multidisciplinary Optimization 1997; 14(1):1-23.

30. Simpson TW, Booker AJ, Ghosh D, Giunta AA, Koch PN, Yang RJ. Approximation methods in multidisciplinary analysis and optimization: a panel discussion. Structural and Multidisciplinary Optimization 2004; 27(5):302-313.

31. Walters FS, Parker Jr LR, Morgan SL, Deming SN. Sequential Simplex Optimization for Quality \& Productivity in Research, Development, and Manufacturing. CRC Press: FL, 1991.

32. Seber GAF, Alan JL. Linear Regression Analysis. Wiley Interscience: NJ, 2003.

33. Mao H, Zhang L, Yang KH, King Al. Application of a finite element model of the brain to study traumatic brain injury mechanisms in the rat. Stapp Car Crash Journal 2006; 50:583-600.

\section{Authors' biographies}

Adel Alaeddini, is a post-doctoral scholar in the Department of Industrial and Operations Engineering at the University of Michigan, Ann Arbor, MI, USA. His research interests include statistical modeling, global optimization, and healthcare operations management.

Kai Yang is a professor in the Department of Industrial and Systems Engineering at Wayne State University, DetroitMI, USA. His field of expertise includes quality and reliability engineering and management, healthcare systems engineering, and product development.

Alper Murat is an assistant professor in the Department of Industrial and Systems Engineering at Wayne State University, Detroit, MI, USA. His research interests include supply chain management, product development, healthcare systems engineering, and data mining. 